\title{
An armed assailant in our hospital: Are we prepared?
}

Sohil Pothiawala ${ }^{1}{ }_{F A M S(E M)}$, Rabind $\underline{\text { Charles }}{ }^{1}{ }_{F A M S(E M)}$, Wai Kein $\underline{\text { Chow }}^{2}$, Kheng Wee $\underline{A n g}^{2}$, Karen Hsien Ling $\underline{\operatorname{Tan}}^{3}$, Mohan $\underline{\text { Tiru }}{ }^{1}$ FAMS (EM)

\begin{abstract}
While armed assailant attacks are rare in the hospital setting, they pose a potential risk to healthcare staff, patients, visitors and the infrastructure. Singapore hospitals have well-developed disaster plans to respond to a mass casualty incident occurring outside the hospital. However, lack of an armed assailant incident response plan can significantly reduce the hospital's ability to appropriately respond to such an incident. The authors describe various strategies that can be adopted in the development of an armed assailant incident response plan. Regular staff training will increase staff resilience and capability to respond to a potential threat in the future. The aim of this article is to highlight the need for the emergency preparedness units of all hospitals to work together with various stakeholders to develop an armed assailant incident response plan. This will be of great benefit for keeping healthcare facilities safe, both for staff as well as for the community.
\end{abstract}

Ann Acad Med Singap 2021;50:712-6

Keywords: Armed assailant, hospital, preparedness, response, strategies

Armed assailant attack in a healthcare facility poses a unique risk to patients, staff and infrastructure alike. According to the US Department of Homeland Security, an armed assailant incident is defined as one where an individual is actively engaged in killing or attempting to kill people in a confined and populated area. ${ }^{1}$

The incidence of armed assailant attacks has been increasing in the US and other parts of the world., ${ }^{2,3}$ Within the healthcare facility, attacks in the emergency department (ED) accounted for roughly one-third of healthcare facility incidents. ${ }^{2}$ While these events are relatively rare in Singapore, the Singapore Terrorism Threat Assessment Report by the Ministry of Home Affairs dated 23 June 2021 continues to classify the country's terrorism threat as high; and warned that Singapore remains vulnerable to attacks against soft targets by lone actors using available objects. ${ }^{4}$ Although there have not been any reported terrorist attacks on Singapore EDs so far, there have been incidents where healthcare staff in the ED have been threatened by patients using various objects such as penknife. In 2015, a police officer was injured in a shooting incident at the
ED of Khoo Teck Puat Hospital. A suspect brought in by the police to seek medical care allegedly grabbed the policeman's gun and 3 shots were fired during the struggle. In 2002, a knife-wielding motorcycle thief was shot by the police when he charged at officers in the car park of Mount Alvernia hospital. A gang-related incident in the ED of Singapore General Hospital saw some people with parangs (machetes) attack another person.

ED is vulnerable to armed assailants as it is a public entry portal to the hospital that allows easy and 24-hour accessibility. Targeting the ED can lead to potentially higher casualties and delay in the care of existing patients. Healthcare institutions in general incorporate multiple buildings, offices, parking areas and other facilities, as well as integrate with the surrounding community and public transport facilities. Such openness makes healthcare organisations vulnerable to external incursions.

In the past, all EDs of public hospitals in Singapore housed a police post manned around-the-clock. However, this police presence has since been withdrawn and

\footnotetext{
${ }^{1}$ Department of Emergency Medicine, Woodlands Health Campus, Singapore

${ }^{2}$ Emergency Preparedness Unit, Woodlands Health Campus, Singapore

${ }^{3}$ Clinical Operations, Woodlands Health Campus, Singapore

Correspondence: Dr Sohil Pothiawala, Department of Emergency Medicine, Woodlands Health, 2 Yishun Central 2, Singapore 768024.

Email: sohil_pothiawala@whc.sg
} 
outsourced to security agencies. The security personnel are stationed not just in the ED, but also other areas of the hospital. The potential threat from an armed assailant can occur in any part of the hospital where these officers may not be present at a particular time. Lack of a dedicated response protocol to an armed assailant, both in the ED and hospital premises, can significantly impact and reduce the hospital's resilience to appropriately handle such an incident. This article aims to highlight the need for all hospitals to work together with various internal and external stakeholders to develop an armed assailant incident response plan.

Hospitals have well-developed disaster plans to respond to mass casualty incidents (MCI) occurring outside the hospital environment. Most hospital staff receive emergency preparedness training to respond to $\mathrm{MCI}$ as part of their job scope, either through online modules or participation in disaster drills. However, an armed assailant in the ED or anywhere in the hospital poses a unique problem as existing plans may not be effective.

The random, short-lasting but violent nature of an armed assailant incident makes healthcare response extremely challenging. ${ }^{3}$ Besides dealing with the assailant, managing the safety of the public and healthcare staff must be paramount during the incident. Because of the dynamic and unpredictable nature of an attack, there is no single best practice. The primary objectives of a response plan are:

- threat assessment and pre-incident planning,

- initiating immediate response during an incident to minimise the assailant's access to potential victims and damage, and

- post-incident recovery and support plans.

\section{Pre-incident planning}

At the very onset, assessment of threat of an armed assailant in a healthcare facility should be carried out, followed by development of a response action plan that establishes the objectives and courses of action. The plan should have input from various stakeholders, including hospital leadership, emergency preparedness unit, medical and nursing staff, security, facility engineering, human resources, law enforcement agencies and legal team. The security of the workplace can be strengthened by adopting a multitiered approach:

- Develop - proactive protective security posture

- Deter - using physical and electronic security measures

- Detect - using alert and visual detection systems
- Delay - putting up measures in place to limit movement of the offender

- Deny - Access to potential victims

Besides incorporating these aspects, an effective response plan should include:

- Data gathering and situational assessment.

- Consideration of security screening system at entrance.

- Closed-circuit television (CCTV) systems connected to the security office for surveillance.

- Restricting access by ensuring appropriate staff identification and areas being accessible with keypad coded doors.

- Early identification of threat by staff.

- Emergency alert notification to Hospital Security Command Centre as well as Singapore Police Force (SPF).

- Lockdown decision matrix for individual departments and buildings.

- Evacuation policy and procedure for patients, visitor and staff; including escape routes and safe areas marked on floor plans.

- Select effective shelter locations for those unable to escape.

- Prior coordination and sharing of plans with first responders and SPF.

\section{During the incident}

\section{Initial assessment and action to an armed assailant situation}

The first challenge is to change the healthcare staff's perspective from ignoring the possibility of such an event to proactively increasing individual awareness and being ready to respond to this situation. ${ }^{5}$ An effective response plan enables the staff to overcome this denial and respond immediately. Staff need to be trained to be more aware of their surroundings, and identify individuals who pose a potential risk in committing a violent act. They must be empowered to immediately raise their concern to hospital security and the police, if required. Banks have alarm buttons behind counters to activate emergency response in the event of a bank robbery. Similarly, hospitals may want to consider installation of similar silent alarm buttons that contact the hospital security centre or SPF. Thus, if an armed assault is in progress, the staff can rapidly activate the emergency notification alert system to help save lives by keeping people out of harm's way, as 
well as triggering a rapid response by the various stakeholders involved. Timely information is crucial, so staff should be trained to contact the police and convey key information such as the location of incident, description and number of attacker(s), type of weapons and other details.

There must be a plan in place so that the public announcement system can inform the building occupants of a potential security threat. This message must also be broadcasted to all security personnel using dedicated communication channels. The emergency notification system should also be equipped to alert people at remote locations within the hospital building or campus, advising them to keep away from the areas under threat. Hospitals can also work with SPF to use the SMS Public Alert System (number 71250) launched by the Ministry of Home Affairs in 2016, which can immediately send one-way SMS to all mobile phone users in the vicinity of this emergency.

All hospitals in Singapore have CCTV systems at various locations. These devices may also be installed at various entry/exit points, perimeter and other critical areas of the hospital to provide live feed for such threats. The system should be connected to the hospital's main security office to enable round-the-clock monitoring and surveillance. The person in charge of monitoring the CCTVs should keep building occupants and security officers updated on the movement and location of the attacker(s) by using the public announcement system and dedicated messaging services, respectively.

Upon activation of alert, there must be a centrally controlled dynamic lockdown matrix for individual areas of the hospital, including lifts and care areas. This approach will help contain the armed assailant within a limited zone, allowing other people in that building to evacuate. Non-impacted zones such as the waiting areas and cafeterias should be evacuated. Plan should also entail a detailed evacuation policy made known to all staff, with escape routes, floor plans and safe areas. A backup evacuation route and location must be planned in case the primary route cannot be used due to assailant presence.

\section{Immediate response model}

According to the law enforcement agencies in US, the response model with an effective impact to protect people during these events has been labelled as "Avoid, Deny, Defend" (also known as "Run, Hide, Fight"). ${ }^{6}$

- Run: The first response is to run away from the assailant, evacuate the area, and once safe, call local law enforcement agency.
- Hide: If the staff and/or patients cannot be evacuated, they should find a place to hide, turn off light and mobile devices and remain quiet. They should lock the door and use nearby equipment and furniture to reinforce the barricade, as this will deny access to the assailant.

- Fight: As a last resort, if there is an imminent danger to life, they can attempt to incapacitate the assailant by using physical aggression, either alone or with team members, or using items that can serve as an improvised weapon.

The Australia-New Zealand Counter Terrorism Committee (ANZCTC) has proposed the model of "Escape, Hide and Tell". ${ }^{7}$ Ontario Hospital Association, Canada has also adopted a similar model of "Evacuate, Hide, Survive and Call". ${ }^{8}$ SGSecure, Singapore's community response to the threat of terrorism, has a similar response model advisory-"Run, Hide, Tell". ${ }^{9}$

A higher value has been placed on providing security agencies with intelligence to improve efficiency and effectiveness of response. Hence, the "Fight" tactic in the US model has been modified to "Tell", similar to that in the Australian and Canadian systems. Considering the closer proximity of security agencies around Singapore, provision of intelligence either by calling SPF at 999, SMS 71999 if unable to speak, or submitting information via SGSecure app is emphasised. Specific silent communication mechanism through the SGSecure app has also been developed to allow informants (affected people of the armed attack in this scenario) to provide secured, silent messages to the response agencies directly.

The adapted model is useful in a setting where staff, patients and visitors can follow the steps of action, especially the "Run" component. However, in an ED or certain areas of the hospital such as intensive care unit, operating/procedure rooms and wards, there are patients who are not mobile or are physically impaired. Many patients are also bed-bound, on life-support equipment or undergoing procedures/surgery. ${ }^{10}$

Healthcare staff must always consider the well-being and safety of their patients and visitors first. During an armed assailant incident, the crisis may overshadow the need to prioritise their own safety. Hence, Inaba et al. suggested an alternate strategy of "Secure, Preserve, Fight" that can be adopted by the healthcare sector to address these concerns. ${ }^{11}$

- Secure: For the safety of patients who cannot be evacuated due to the aforementioned reasons, and also the staff who need to be with them, the location 
should be promptly secured. Locking mechanism should be immediately activated by the respective areas to secure their entrances, so that the assailant is unable to enter those zones.

- Preserve: Healthcare staff taking care of these patients should continue providing ongoing care to preserve life.

- Fight: This strategy should be used only if absolutely necessary, as stated before.

However, for the Singapore context, it would be more appropriate to adapt the "Fight" component to the "Tell" tactic. Rarely, there may be a need to consider incapacitating the armed assailant in the event they are identified. But this should only be undertaken as a last resort to protect life.

Thus, based on this best available evidence and in line with the national initiative of SGSecure to counter terrorism, adopting the model of "Run, Hide, Tell" or "Secure, Preserve, Tell" is recommended. This needs to be individualised, taking into consideration the infrastructure, department and types of patients. Adopting strategies that would best suit Singapore's needs can significantly reduce the risks to patients and staff.

Healthcare workers (HCWs) in the proximity of the armed assailant should not attempt to engage the assailant in any way, whether verbal and/or physical. Trained security personnel can attempt to de-escalate the situation by maintaining adequate distance from the attacker and not try to apprehend or overpower the assailant. They can continue talking and engaging the assailant and buy time where possible.

Another key concept that needs to be a part of the response model is "Treat". Being in a healthcare setting, this is intuitive for any response. Once the scene is safe and the assailant has been neutralised, the priority is to identify seriously wounded casualties, patients or staff, who require immediate care for injuries. For someone who is bleeding profusely while hiding, all staff should be trained to identify and treat airway obstruction and major arterial haemorrhage by application of direct pressure or a tourniquet. Healthcare facilities should ensure they have adequate tourniquets to respond to an armed assailant incident.

Care provided during an armed assailant event should be remembered by the acronym THREAT: ${ }^{12}$

$\mathrm{T}$ - Threat suppression

$\mathrm{H}$ - Haemorrhage control

RE - Rapid Extrication to safety

A - Assessment by medical providers

$\mathrm{T}$ - Transport to definitive care

\section{Management of public and media during the incident}

In the current era of social media, news of an incident will travel fast, resulting in influx of media personnel, family and friends of patients and staff, as well as curious bystanders. Anticipating this, a preidentified area some distance from the hospital should be cordoned off by the security to ensure public safety.

\section{Arrival of law enforcement officers}

When armed officers from the SPF or the Special Tactics and Rescue (STAR) team (a tactical unit of SPF) arrive, the security in-charge should quickly liaise with them at a designated meet-up point and brief them about the current details of the incident, precautionary actions taken, and review floor plans and CCTV images as necessary. Staff and visitors should remain calm and strictly follow the enforcement team's instructions. They should not ask the officers for help, leading to delay or impeding the latter's movement as their primary aim is to neutralise the threat. People should only exit the area when instructed to do so by the police or security officers. Upon neutralisation of the threat and after advice from SPF, an "All Clear" message should be broadcasted by security across the hospital.

\section{Post-incident}

\section{Recovery and business continuity}

Once the attack has ended and the threat has been neutralised, there is a need to assess the ability of the department or hospital to continue routine operations. Certain operations may be affected when that particular area is secured for investigation, or suffers damage to facilities and equipment. Those in charge must determine if the department retains the capacity and staff to treat its existing patients and new casualties, or an alternate treatment area at a different location needs to be set up to resume operations.

\section{Behavioural health support plan}

In the immediate aftermath of an attack, HCWs, patients and other witnesses may be "secondary victims" due to physical or mental trauma. As part of the recovery process, the hospital needs to consider providing rapid psychological assessment and first aid to the affected people as necessary. All HCWs from the affected areas should undergo a debriefing session from their supervisors before resuming work. Their particular needs or concerns should be appropriately addressed, with those affected provided support by peers or medical social workers. Such support, after the incident and also as outpatient follow-up, should be arranged for all 
people affected by the event, to reduce the traumatic impact as well as to ease their recovery.

\section{After-action review}

A review should be conducted after an incident in a timely manner, as valuable insights can be obtained during this process. Improvement of response plan is the goal of any preparedness effort. If the response plan is modified, the new plan should be retested to assure that it improves the entire system's capability to respond.

\section{Preparedness to an armed assailant attack}

Every hospital should develop a response plan to effectively address this risk. Valuable input from internal and external stakeholders such as SPF, Singapore Civil Defence Force (SCDF), Certis Cisco security and Ministry of Health should be obtained while devising these plans. Familiarisation of the response plan, supplemented by periodic training of all healthcare staff, will play an important role in triggering an initial response, as well as adopting the subsequent appropriate steps to mount an effective and efficient response. The training module of the response plan, including videos, should be developed and made available on the organisation's intranet for periodic training.

The next step would be to conduct table-top as well as full-scale armed assailant mock drill involving healthcare staff and volunteers participating as an armed assailant and patients. Officers from SPF, SCDF and STAR teams should come to the hospital in full gear to respond to this mock event. These exercises are a great way to evaluate the preparedness plan, clarify roles and responsibilities, provide training, improve coordination between internal and external stakeholders, obtain participant feedback, and identify deficiencies and recommendations for plan improvement. The lessons learnt from these joint exercises would be invaluable to instil confidence and improve readiness for such incidents. Moreover, with advancement in technology, deployment of CCTV with real-time artificial intelligence video surveillance, facial recognition at entry points, and use of robots to help security scale up patrols without manpower resourcing can also be adopted for surveillance and deterrence of such attacks.
In summary, armed assailant events in the hospital are rare, and it is challenging to predict their occurrence. The emergency preparedness units of all hospitals should work together, along with various stakeholders, especially SPF and SCDF, to develop hospital-specific armed assailant incident response plan. Regular staff training, incorporating online learning modules and simulated drills, will increase the resilience and capability of hospitals to respond to a potential threat in the future. This would be of immense benefit to keep healthcare facilities safe, both for staff as well as for the community.

\section{REFERENCES}

1. US Department of Health and Human Services. Incorporating Active Shooter Planning into Health Care Facility Emergency Operations Plans, 2014. Available at: http://www.phe.gov/ preparedness/planning/Documents/active-shooter-planning-eop2014. pdf. Accessed 12 June 2021.

2. Kelen GD, Catlett CL, Kubit JG, et al. Hospital-based shootings in the United States: 2000 to 2011. Ann Emerg Med 2012;60:790-8.

3. Goralnick E, Walls R. An active shooter in our hospital. Lancet 2015;385:1728.

4. Ministry of Home Affairs. Singapore Terrorism Threat Assessment Report 2021, 23 June 2021. Available at: https://www.mha.gov.sg/ mediaroom/press-releases/singapore-terrorism-threat-assessmentreport-2021/. Accessed on 15 July 2021.

5. Rorie S. Implementing an active shooter training program. AORN J 2015;101:C5-6.

6. Advanced Law Enforcement Rapid Response Training (ALERRT) Center at Texas State University. Avoid deny defend. Available at: https://www.avoiddenydefend.org/ Accessed on 12 June 2021.

7. Australia-New Zealand Counter-Terrorism Committee. Active Armed offender guidelines for crowded places, 2017. Available at: https://www.nationalsecurity.gov.au/Media-and-publications/ Publications/Documents/active-armed-offender-guidelines-crowdedplaces.pdf. Accessed on 12 July 2021.

8. Ontario Hospital Association. Code Silver: Person with a weapon, 2019. Available at: https://www.oha.com/Documents/Code\%20 Silver\%20Development\%20Guidance.pdf. Accessed on 12 July 2021.

9. SGSecure. Available at: https://www.sgsecure.gov.sg/. Accessed on 12 July 2021

10. Gerold KB. Hospital Planning and Response to an Active Shooter Incident: Preparing for the $\mathrm{n}=1$. Anesthesiol Clin 2019;37:161-9.

11. Inaba K, Eastman AL, Jacobs LM, et al. Active-shooter response at a health care facility. N Engl J Med 2018;379:583-6.

12. Healthcare \& Public Health Sector Coordinating Council. Active shooter planning and response. International Association of Emergency Medical Services Chiefs, 2017. 